Artículo original

(Original paper)

\title{
HELMINTOFAUNA DEL APARATO DIGESTIVO Y RESPIRATORIO DE AVES ACUÁTICAS (ANATIDAE: ANATINAE) DEL HUMEDAL DE ATLANGATEPEC, TLAXCALA, MÉXICO
}

\author{
HELMINTH FAUNA FROM THE DIGESTIVE AND RESPIRATORY \\ SYSTEMS OF WATERFOWL (SUBFAMILY: ANATINAE) \\ AT WETLAND OF ATLANGATEPEC, TLAXCALA MEXICO
}

\section{Patricia PAdIlla-AGUILAR, ${ }^{1, *}$ David OSORIO-SARABIA, ${ }^{2}$ Claudia I. MUÑOZ-GaRCÍA, ${ }^{3}$ EMILIO RENDÓN-FRANCO ${ }^{3}$ Y EVANGELINA ROMERO-CALLEJAS ${ }^{1}$}

\author{
${ }^{1}$ Laboratorio de Diagnóstico de Parasitología, Facultad de Medicina Veterinaria y Zootecnia, Universidad \\ Nacional Autónoma de México. < mvz_patypadilla@yahoo.com >; <eva_romeroc@yahoo.com.mx > \\ ${ }^{2}$ Laboratorio de Helmintología, Instituto de Biología, Universidad Nacional Autónoma de México. \\ <davidos1947@hotmail.com> \\ ${ }^{3}$ Departamento de Producción Agrícola y Animal, Universidad Autónoma Metropolitana Unidad Xochimilco \\ <chiqui_mex@yahoo.com>; <erendonfranco@yahoo.es> \\ *Autor para correspondencia: <mvz_patypadilla@yahoo.com> \\ Recibido: 14/06/2017; aceptado: 11/12/2017; publicado en línea: 16/03/2018 \\ Editor responsable: Octavio Rojas Soto
}

Padilla-Aguilar, P., Osorio-Sarabia, D., Muñoz-García, C. I., Rendón-Franco, E. y Romero-Callejas, E. (2018) Helmintofauna del aparato digestivo y respiratorio de aves acuáticas (Anatidae: Anatinae) del humedal de Atlangatepec, Tlaxcala, México. Acta Zoológica Mexicana (n.s), 34(1), 1-5.

RESUMEN. El objetivo de esta investigación fue realizar el primer inventario de helmintos recuperados a partir del tubo digestivo y del aparato respiratorio de 52 aves acuáticas de la familia Anatidae, procedentes del humedal de Atlangatepec, Tlaxcala, a partir del aparato digestivo y respiratorio, los cuales fueron donados por cazadores en la temporada de caza de noviembre 2012- febrero 2013 y noviembre 2013- febrero 2014. Los parásitos encontrados se procesaron empleando técnicas convencionales para el examen helmintológico. Se identificaron 21 taxa de helmintos: siete trematodos (Cotylurus. cornutus, Australapatemon burti, Zygocotyle. lunata, Notocotylus. seineti, Typhlocoelum. sisowi, Echinostoma sp. y Echinoparyphium recurvatum.), cuatro cestodos (Hymenolepis sp., Cloacotaenia megalops, Choanotaenia infundibulum y Fimbriaria fasciolaris), ocho nematodos (Amidostomum sp., Streptocara sp., Epomidiostomum uncinatum ,Syphaciella sp., Capillaria sp., Tetrameres. fissispina, Tetrameres sp. y Echinuria uncinata) y dos acantocéfalos (Pseudocorynosoma constritum y Polimorphus sp.), entre ellos destaca Syphaciella sp., ya que se registra por primera vez para México. También se registra por primera vez para México a los trematodos: Z. lunata y $A$. burti en $A$. crecca y $A$. strepera. En el caso de los cestodos a $C$. infundibulum en A. affinis.

Palabras clave: helmintos, aves acuáticas, anátidos, Atlangatepec, patos.
Padilla-Aguilar, P., Osorio-Sarabia, D., Muñoz-García, C. I., Rendón-Franco, E., \& Romero-Callejas, E. (2018) Helminth fauna from digestive and respiratory tract of waterfowl (Subfamily: Anatinae) at wetland of Atlangatepec, Tlaxcala Mexico. Acta Zoológica Mexicana (n.s), 34(1), 1-5.

ABSTRACT. The purpose of this investigation was to carry out the first inventory of helminths collected from waterfowl $(n=52)$ of the family Anatidae. This work took place in wetland Atlangatepec, Tlaxcala. Helminths were collected from the digestive tract and the respiratory system of waterfowl donated by hunters. Collected parasites were processed using conventional fixation and staining techniques for the identification of helminth. Twenty-one taxa of helminth swere identified as follow: seven trematodes (Cotylurus cornutus, Australapatemon burti, Zygocotyle lunata, Notocotylus seineti, Typhlocoelum sisowi, Echinostoma sp. and Echinoparyphium recurvatum), four cestodes (Hymenolepis sp, Cloacotaenia megalops, Choanotaenia infundibulum and Fimbriaria fasciolaris), eight nematodes (Amidostomum sp., Streptocara sp., Epomidiostomum uncinatum, Syphaciella sp., Capillaria sp., Tetrameres fissispina, Tetrameres sp. and Echinuria uncinata) and two Acanthocephala (Pseudocorynosoma constritum and Polimorphus sp.). It is relevant to highlight that this is the first report of Syphaciella sp. in waterfowl in Mexico.

Key words: Helminth, waterfowl, duck, Atlangatepec, nematodes. 


\section{INTRODUCCIÓN}

Los helmintos son muy abundantes en la naturaleza y son un grupo polifilético, ya que bajo este término se incluyen representantes de diferentes phyla que no están relacionados filogenéticamente: Platyhelminthes, Nematoda, Acanthocephala y Annelida (Pérez-Ponce de León \& García-Prieto, 2001). Algunos de estos parásitos dentro de su ciclo biológico utilizan a las aves acuáticas como huéspedes definitivos (Barrera-Guzmán \& Guillen-Hernández, 2008). Las aves adquieren a los helmintos principalmente por sus hábitos alimenticios, mediante el consumo de huéspedes intermediarios, entre otros factores, por lo que son consideradas como el grupo de vertebrados con mayor riqueza parasitaria (Poulin, 1995).

Los humedales mexicanos reúnen las condiciones ecológicas necesarias para albergar decenas de especies de aves migratorias y residentes, un ejemplo de esto es la presa de Atlangatepec, que fue decretada como hábitat prioritario para aves acuáticas residentes y migratorias en el 2009 (Ramsar list, 2014), uno de los anátidos endémicos de México que habita este lugar es el Pato Mexicano (Anas diazi) que se encuentra en el listado de la NOM059-ECOL-2001 como especie amenazada (SEMARNAT, 2002), existe escasa información de esta especie a pesar de su estado de conservación (Pérez et al., 2002). Los estudios sobre los helmintos de este pato son escasos o no están actualizados.

El objetivo de esta investigación fue realizar el primer inventario de helmintos del aparato digestivo y respiratorio de aves acuáticas de la familia Anatidae procedentes del humedal de Atlangatepec, Tlaxcala.

\section{MATERIAL Y MÉTODOS}

Las muestras biológicas fueron obtenidas mediante la actividad cinegética, recolectándose los aparatos digestivos y respiratorios de 52 aves acuáticas (Anas discors, $A$. crecca, A. diazi, A. strepera, A. clypeata y Aythya affinis) durante las temporadas de caza noviembre 2012- febrero 2013 y noviembre 2013- febrero 2014 en la presa de Atlanatepec ( $\mathrm{N} 19^{\circ} 33.596^{\prime}$ y O $\left.098^{\circ} 11.368^{\prime}\right)$, ubicada en el estado de Tlaxcala, México.

Para la revisión helmintológica los aparatos digestivos y respiratorios fueron colocados en cajas de Petri con solución salina al $0.85 \%$, posteriormente se examinaron bajo el microscopio estereoscópico. Lo anterior se realizó en el Laboratorio de diagnóstico Parasitológico de la Fa- cultad de Medicina Veterinaria y Zootecnia (FMVZ) de la Universidad Nacional Autónoma de México (UNAM).

Los parásitos encontrados en del aparato gastrointestinal y respiratorio fueron extraídos con un pincel delgado contados in situ; trematodos, acantocéfalos y cestodos se fijaron en formol al $4 \%$ caliente, mientras que los nematodos en alcohol salino al 4\% caliente; finalmente, todos los helmintos se conservaron en alcohol 70\% (Lamothe, 1997); los platelmintos y acantocéfalos fueron teñidos con hematoxilina de Delafiel o paracarmin de Meyer, los nematodos fueron transparentados con lactofenol de Amman o glicerol-alcohol (1:1). Lo anterior se realizó en el Laboratorio de Helmintología del Instituto de Biología de la UNAM.

Los helmintos fueron observados, medidos y dibujados bajo el microscopio óptico para su identificación dando énfasis en las estructuras características para su determinación taxonómica, lo anterior se realizó utilizando claves y literatura especializada como: Yamaguti (1940), McDonald (1981 \& 1988), Czaplinzki y Vaucher (1994), Gibson et al. (2002) y Anderson et al. (2009).

Los ejemplares de referencia fueron depositados en el Laboratorio de diagnóstico Parasitológico de la FMVZ de la UNAM y en la Colección Nacional de Helmintos entre los números de catálogo 9713 a 9724.

Los niveles de infección fueron determinados con los parámetros ecológicos propuestos por Bush et al. (1997).

\section{RESULTADOS}

De las 52 aves acuáticas examinadas 39 (75\%) resultaron positivas a algún tipo de helminto. Se obtuvo una riqueza de 21 taxa (Cuadro 1): siete trematodos (C. cornutus, $A$. burti, Z. lunata, N. seineti T. sisowi, Echinostoma sp. y E. recurvatum.), cuatro cestodos (Hymenolepis spp., $C$. megalops, C. infundibulum y F. fasciolaris), ocho nematodos (Amidostomum sp., Streptocara sp., E. uncinatum, Syphaciella sp., Capillaria sp., T. fissispina, Tetrameres sp. y E. uncinata) y dos acantocéfalos ( $P$. constritum y Polimorphus sp.). Del total de helmintos recuperados (615) los cestodos representan el 69.9\%, los trematodos el $18.8 \%$, los nematodos el $10.2 \%$ y por último los acantocéfalos con un $0.9 \%$.

\section{DISCUSIÓN}

Esta investigación registra por primera vez 21 taxa (13 especies nominales y 8 identificados a nivel genérico), 
Cuadro 1. Lista de helmintos identificados en aves acuáticas del humedal de Atlangatepec, Tlaxcala.

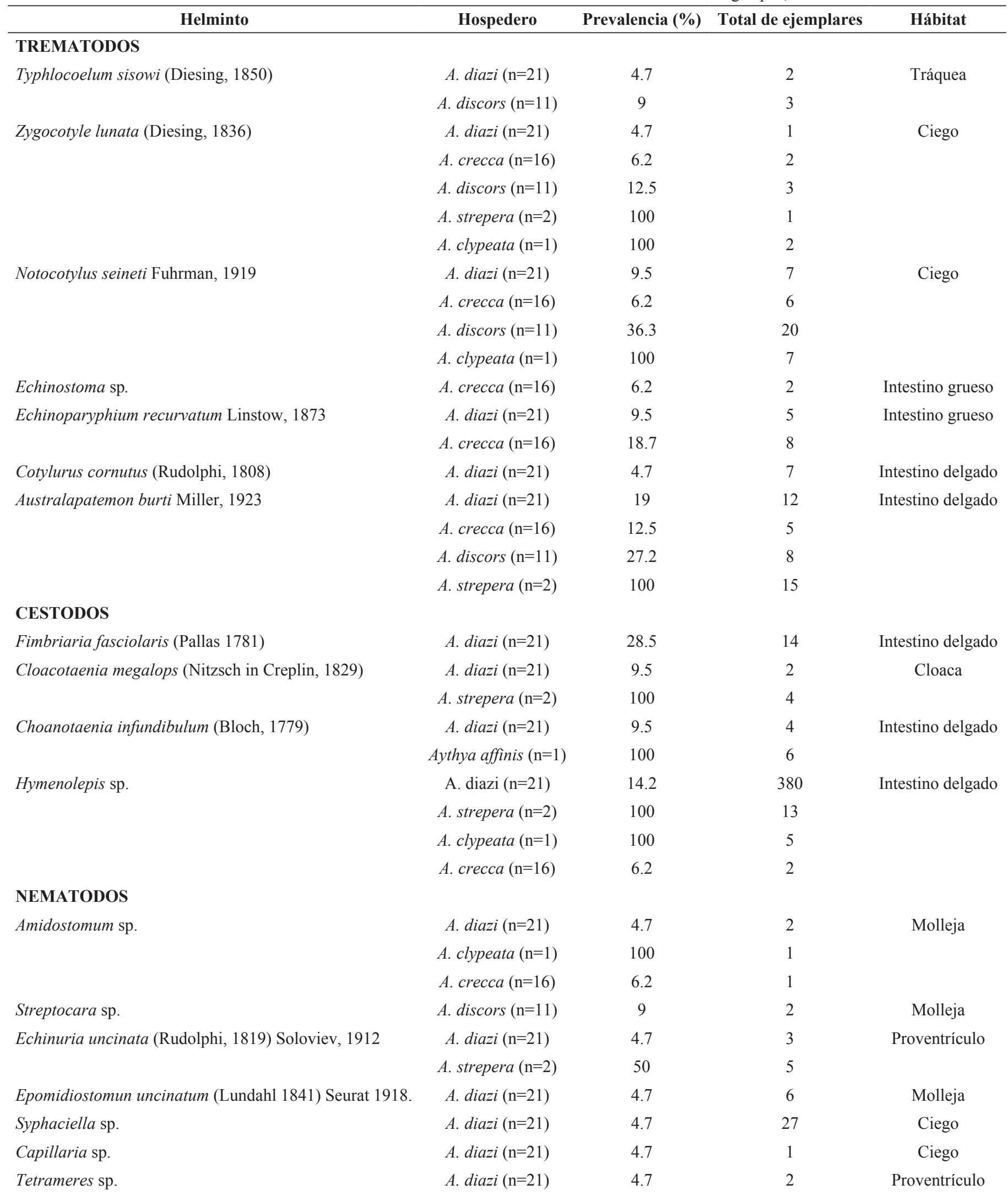


Cuadro 1. Continúa.

\begin{tabular}{|c|c|c|c|c|}
\hline Helminto & Hospedero & Prevalencia (\%) & Total de ejemplares & Hábitat \\
\hline & A. discors $(\mathrm{n}=11)$ & 18 & 6 & \\
\hline & A. $\operatorname{crecc} a(\mathrm{n}=16)$ & 6.2 & 1 & \\
\hline \multicolumn{5}{|l|}{ ACANTOCÉFALOS } \\
\hline \multirow[t]{2}{*}{ Pseudocorynosoma constrictum Van Cleave, 1918} & A. $\operatorname{discors}(\mathrm{n}=11)$ & 9 & 2 & Intestino delgado \\
\hline & A. $\operatorname{diazi}(\mathrm{n}=21)$ & 4.7 & 1 & \\
\hline Polimorphus sp. & A. discors $(\mathrm{n}=11)$ & 9 & 2 & Intestino delgado \\
\hline
\end{tabular}

para el humedal de Atlangatepec, Tlaxcala, México. Entre ellos destaca Syphaciella sp., ya que se registra por primera vez para México. También se registra por primera vez para México a los trematodos: Z. lunata y A. burti en $A$, crecca y $A$. strepera. En el caso de los cestodos a $C$. infundibulum en $A$. affinis.

Anas diazi es nueva especie de hospedero en México para T. sisowi, C. cornutus, C. infundibulum, E. uncinatum, Syphaciella sp. y T. fissispina. La importancia de generar información sobre este anátido es por su estatus que se encuentra en la categoría de amenazado en la NOM059-ECOL-2001 y es una especie considerada endémica de México.

La riqueza obtenida en este trabajo (21 taxa) es similar a la reportada por Martínez-Haro et al. (2012), realizado en aves en las ciénagas de Lerma, Gladden \& Canaris, (2009), realizado en humedales de Chihuahua, con riquezas de 20 y 23 respectivamente. Lo anterior confirma lo señalado por Poulin (1995), quien establece a las aves como el grupo de vertebrados con mayor riqueza parasitaria que existe.

De los huéspedes, el que presentó la mayor riqueza fue A. diazi con 16 especies de helmintos. Lo anterior podría atribuirse a su conducta biológica (pato buceador) y a su dieta, formada por pequeños moluscos y crustáceos, particularmente bivalvos, anfípodos y crustáceos (Leopold, 1959; Johnsgard, 1978), los cuales son utilizados como huéspedes intermediarios por la mayoría de las especies de helmintos identificadas en este pato (Wobeser, 1981; Atkinson et al., 2008).

De los trematodos identificados los del género Notocotylus fueron los que mayor dificultad presentaron para identificar a nivel específico, principalmente por su similitud considerable en las estructuras con importancia diagnóstica (testículos, ovarios, glándulas vitelógenas, metratermo, entre otros) y pocos caracteres efectivos pa- ra su identificación (Herber, 1942; Martínez-Haro et al., 2012).

Entre los cestodos identificados, C. megalops, F. fasciolaris y Choanotaenia infundibulum son muy comunes en aves acuáticas, por lo que resultó de fácil identificación. Lo contrario ocurrió con Hymenolepis sp., en general este grupo de helmintos son difíciles de identificar debido a que son muy frágiles y difíciles en su manejo, ya que durante su procesamiento puede haber una pérdida de sus ganchos al ser estructuras muy delicadas o se pueden fragmentar, lo que impide una adecuada determinación taxonómica (Czaplinski \& Vaucher, 1994).

De los nematodos identificados en la mayoría solo se llegó hasta género; lo anterior se debe principalmente a que se encontraron únicamente hembras, lo que puede ser explicado porque los machos son más pequeños, por ejemplo en $T$. fissispina se localizan en el lumen del proventrículo, mientras que las hembras se encuentran embebidas en las glándulas, de ahí que éstas son fáciles de observar, mientras que los machos necesitan ser buscados en el moco (Martínez-Carrasco et al., 2009).

El parasitismo es una interacción común en las aves, por tanto estos organismos han coevolucionado con algunas especies parásitos por lo que casi no les generan daños; sin embargo, factores como la contaminación y la reducción del hábitat entre otros, pueden ocasionar que este tipo de enfermedades rompan un equilibrio y generen muertes masivas de aves acuáticas, de ahí la importancia de continuar con estos trabajos a largo plazo para poder elaborar programas de manejo para la conservación de estos organismos (Wobeser, 1981; Atkinson et al., 2008).

AGRADECIMIENTOS. Al MVZ Julio García Hernández, MVZ Leonardo Mata Abrego, Bióloga Maritza Alexandra Ramírez y MVZ Isabel Guadarrama Gonzaga por el apoyo recibido en el Laboratorio de Diagnóstico Parasitológico de la Facultad de Medicina Veterinaria y Zootecnia de la Universidad Nacional Autónoma de México. 


\section{LITERATURA CITADA}

Anderson, R., Chabaud A. \& Willmott, S. (1986) Keys to the nematode parasites of vertebrates. Oxford University Press, New York. $480 \mathrm{pp}$.

Atkinson, C. T., Thomas, J. N. \& Bruce, D. H. (2008) Parasitic diseases of wild birds. U.S.A. Wiley-Blackwell. 3-11 p.

Barrera-Guzmán, A. \& Guillen-Hernández, S. (2008) Helmintos intestinales en aves Ciconiformes de la ciénega de Chuburná, Yucatán, México. Revista Mexicana de Biodiversidad, 79, 525-527.

Bush, A. O., Lafferty, K. D., Lotz, J. L \&. Shostak, A. W. (1997) Parasitology meets ecology on its own terms: Margolis et al. revisited. Journal of Parasitology, Washington, D.C., 83, 575-583.

Czaplinski, B. \& Vaucher, C. (1994) Family Hymenolepididae Ariola, 1899. Pp. 595-663. In: L. F. Khalil, A. Jones and R. A. Bray (eds.) Keys to the cestode parasites of vertebrates, Commonwealth Agriculture Bureaux International, Wallingford.

Gibson, I. D., A. Jones \& Bray, R. A. (2002) Keys to the Trematoda, vol 1. CABI/ The Natural History Museum, London. 521 pp.

Gladden, B. W. \& Canaris, A. G. (2009) Helminth parasites of the Bufflehead duck, Bucephala albeola, wintering in the Chihuahua desert with a checklist of helminth parasites reported from this host. Journal Parasitology, 95, 129-136.

Herber, E. C. (1942) Life history studies on two trematodes of the subfamily Notocotylinae. The Journal of Parasitology, 28, 179196.

Johnsgard, P. A. (1978) Ducks, geese, and swans of the world. University of Nebraska Press, Lincoln. 404 pp.

Lamothe A. R. (1997) Manual de técnicas para preparar y estudiar los parásitos de animales silvestres. AGT Editor, México D. F. $43 \mathrm{pp}$.

Leopold, S. (1959) Wildlife of Mexico. Berkley, C. A. University of California Press. 37 pp.

Martínez-Carrasco, C. M., Ruiz de Ybáñez, M., Espigares, D., Tizzani, P. \& Alonso, F. D. (2009) Tetrameriosis in feral pigeons from Murcia, Southeastern Spain. Preventive Veterinary Medicine, 90, 284-286.

Martínez-Haro, M., Sánchez-Nava, P., Salgado-Maldonado G. \& Rodríguez-Romero, F. J. (2012) Helmintos gastrointestinales en aves acuáticas de la subcuenca alta del río Lerma, México. Revista Mexicana de Biodiversidad, 83, 36-41.

McDonald, M. E. (1981) Key to trematodes reported in waterfowl. US Department of the Interior Fish and Wildlife Service, Washington, D. C. 17-156 pp.

McDonald, M. E. (1988) Key to Acanthocephala reported in waterfowl. US Department of the Interior Fish and Wildlife Service, Washington, D. C. 23 pp.

Pérez Ponce de León, G. \& García-Prieto, L. (2001) Diversidad de helmintos parásitos de vertebrados silvestres de México. CONABIO. Biodiversitas, 37:7-11.

Pérez, A. A., Gaston, K. J. \& Kershaw, W. M. (2002) Populations trends and priority conservation sites for Mexican duck Anas diazi. Bird Conservation International, 12, 35-52.

Poulin, R. (1995) Phylogeny, ecology, and the richness of parasite communites in vertebrales. Ecological Monographs, 65 (3), 283302 .

Ramsar list. (2014) The list of wetlands of international importance. Convention on Wetlands of Internacional Importance (Ramsar, Iran, 1971). Ramsar Convention, Gland. 1-38 pp.

SEMARNAT (Secretaría de Medio Ambiente y Recursos Naturales). (2002) Norma Oficial Mexicana NOM-059-ECOL-2001. Protección ambiental-Especies nativas de México de flora y fauna silvestres-Categorías de riesgo y especificaciones para su inclusión, exclusión o cambio-Lista de especies en riesgo. Diario Oficial de la Federación, 6 de marzo de 2002, México, D.F. 1-85 pp.

Wobeser, G. A. (1981) Diseases of Wild Waterfowl. New York, United States of America, Plenum Press.129-146 pp.

Yamaguti, S. (1940) Studes of the helmint fauna of Japan. Part 3. Cestodes of birds II. Japanese Journal of Medical Sciences VI. Bacteriology and Parasitology, 1, 175-211. 